\title{
Electrodynamic balance measurements of thermodynamic, kinetic, and optical aerosol properties inaccessible to bulk methods
}

\author{
S. S. Steimer ${ }^{1,2}$, U. K. Krieger ${ }^{2}$, Y.-F. Te ${ }^{2}$, D. M. Lienhard ${ }^{2, *}$, A. J. Huisman ${ }^{2,3}$, B. P. Luo ${ }^{4,2}$, M. Ammann ${ }^{1}$, and \\ T. Peter ${ }^{2}$ \\ ${ }^{1}$ Laboratory of Radiochemistry and Environmental Chemistry, Paul Scherrer Institute, 5232 Villigen PSI, Switzerland \\ ${ }^{2}$ Institute for Atmospheric and Climate Science, ETH Zurich, 8092 Zurich, Switzerland \\ ${ }^{3}$ Chemistry Department, Union College, Schenectady, New York 12308, USA \\ ${ }^{4}$ Physikalisch-Meteorologisches Observatorium Davos and World Radiation Center PMOD/WRC, Davos, Switzerland \\ *now at: Department of Chemistry, University of Cambridge, Cambridge, UK
}

Correspondence to: S. S. Steimer (sarah.steimer@psi.ch) and U. K. Krieger (ulrich.krieger@env.ethz.ch)

Received: 13 December 2014 - Published in Atmos. Meas. Tech. Discuss.: 20 January 2015

Revised: 29 April 2015 - Accepted: 10 May 2015 - Published: 8 June 2015

\begin{abstract}
Measurements of a single, levitated particle in an electrodynamic balance are an established tool for deriving thermodynamic and material data such as density, refractive index and activities of components of an aqueous solution under supersaturated conditions, where bulk measurements are not possible. The retrieval relies on combining mass-tocharge data and size data from light scattering. Here, we use a combination of low- and high-resolution Mie resonance spectroscopy to obtain radius data, enabling an accurate size determination not only when the particle is in equilibrium, but also when it is out of equilibrium due to kinetic limitation of mass transport. With the data measured under nonequilibrium conditions, it is possible to retrieve the water diffusivity. A challenge is that the radius retrieval by comparing measured light scattering with Mie theory requires the knowledge of refractive index as a function of concentration. Here, we show an iterative retrieval of refractive index and size for compounds for which data cannot be obtained in the bulk either due to lack of sufficient amounts of sample or limited solubility. We demonstrate the measurement strategy and the retrieval of water activity, density, refractive index and water diffusivity for aqueous shikimic acid. Water diffusivity in concentrated shikimic acid decreases by 6 orders of magnitude at $250 \mathrm{~K}$ compared to that at room temperature.
\end{abstract}

\section{Introduction}

In 1935 Millikan stated that his famous oil drop experiment may be used as a very sensitive balance: "This device is simply an electrical balance in place of a mechanical one, and it will weigh accurately and easily to one ten-billionth of a milligram." As early as 1960, Gucker and Rowell reported light scattering experiments on single levitated droplets in a Millikan-type setup. Since then, the techniques to measure charge-to-mass ratio and to size single, levitated droplets using light scattering have considerably matured and have been applied extensively to investigate atmospheric aerosols. For example, Tang and Munkelwitz used light-scattering data of single aqueous salt droplets levitated in an electrodynamic balance (EDB) to determine water activity, density and refractive indices (Tang and Munkelwitz, 1991, 1994). These measurements were made in quasi-thermodynamic equilibrium, whereas measurements of organic aerosol particles are often subject to kinetic limitations of water transport within the condensed phase. They also could take advantage of having enough sample material to perform bulk measurements, which allowed them to tie the experiments on single droplets to those obtained with more traditional techniques. Here we present a measurement and retrieval technique to obtain these data also for systems, which suffer from both: strong water uptake impedance and low solubility and/or limited amount of sample material. 
We will illustrate our approach using shikimic acid as an example of a compound with comparably low solubility in water $\left(180 \mathrm{~g} \mathrm{~L}^{-1}\right.$ at $23^{\circ} \mathrm{C}$ (Budavari, 1996), corresponding to a mole fraction of 0.025 at saturation), so that the water activity of the saturated solution is close to 1 . Shikimic acid is a carboxylic acid which has been detected in biomass burning aerosol (Medeiros and Simoneit, 2008). In recent years it has been shown that atmospheric organic matter can adopt an amorphous solid state (Zobrist et al., 2008; Virtanen et al., 2010). In the case that organic aerosols host chemical reactions, the gradual decrease in diffusivity from liquid to amorphous solid state is likely to cause a reduction in reaction rate, an effect well known for crystalline material (Moise and Rudich, 2002). First investigations of this effect for amorphous material have been made by Shiraiwa et al. (2011), Kuwata and Martin (2012) and Zhou et al. (2013). As we are using the ozonolysis of shikimic acid as a model system to investigate the interplay of physical and chemical kinetics (Steimer et al., 2014), we need a detailed understanding of how relative humidity affects the physical state and diffusivity of water in shikimic acid. Additionally, the dependence of the concentration on water activity has to be known to determine initial bulk phase concentrations of shikimic acid for rate law assessments.

\section{Experimental setup}

The basic setup of the electrodynamic balance (EDB) has been described previously (Krieger et al., 2000; Colberg et al., 2004; Lienhard et al., 2014). Here, we summarize its basic features as follows: the EDB is placed in a doublejacketed glass chamber, with a cooling liquid flowing between the inner walls and an insulation vacuum between the outer walls. The temperature can be adjusted between 180 and $320 \mathrm{~K}$, covering the entire atmospheric range. A gas mixture is pumped continuously through the chamber to adjust/control relative humidity; the total pressure can be varied between 150 and $1000 \mathrm{hPa}$. The EDB is loaded by a single particle generator (Hewlett-Packard 51633A ink jet cartridge) filled with a dilute aqueous solution of the sample material. Relative humidity $(\mathrm{RH})$ is measured by a capacitive $\mathrm{RH}$ probe with an integrated temperature sensor (U.P.S.I., France, model G-TUS.13R) mounted in the upper-end cap of the EDB in close proximity to the levitated particle $(<10 \mathrm{~mm}$ distance). The sensor was calibrated with deliquescence relative humidities of common salts and the saturation vapor pressure over ice at several temperatures. Capacitive RH probes are known to show some hysteresis and we conservatively estimate the accuracy to be no worse than $\pm 3 \%$.

Four independent methods to characterize the aerosol particle are used: (i) the DC voltage applied to compensate the gravitational force is proportional to and used as a measure for the mass of the particle. (ii) The two-dimensional angular scattering pattern is measured over a scattering an- gle ranging from 78 to $101^{\circ}$ and used to estimate the radius of the particle and to detect phase changes (Krieger and Meier, 2011). (iii) Mie resonance spectroscopy with a LED"white"-light source for illumination is used to follow the radius change of a spherical particle (e.g., a liquid droplet); the wavelength of the LED is centered at the sodium D-Line $(589 \mathrm{~nm})$. The back-scattered light from the LED is collected by a spectrograph with a slow scan back-illuminated CCD (charge-coupled device) array detector as an optical multichannel analyzer, for details see (Zardini et al., 2006; Zardini and Krieger, 2009). (iv) High resolution Mie resonance spectroscopy is used to measure size and refractive index simultaneously with high precision as described in more detail below.

The pioneering work of Ashkin and Dziedzic (1977) showed that size and refractive index of spherical particles may be obtained from Mie resonance spectra with very high precision. These Mie resonances, also called whispering gallery modes, have been observed for both elastic (e.g., Chylek et al., 1983; Ray et al., 1991) and inelastic scattering (e.g., Conwell et al., 1984; Preston and Reid, 2013). Conventionally, those spectra have been measured using a fixed wavelength, while drying or humidifying a particle and hence changing its size. In this approach, the Mie parameter, $X$, varies with time $(X(t)=2 \pi r(t) / \lambda$, with $\lambda$ being the wavelength and $r$ being the radius). This method becomes difficult to apply if the rate of size change is small, because several resonances are needed to compare measured spectra with Mie scattering computations. Hence, the technique cannot be applied to compounds which need extended equilibration times because of low water diffusivity.

These problems do not arise when using a wavelength tunable light source. Huckaby et al. (1994) achieved relative errors in radius and refractive index of $3 \times 10^{-5}$ and determined dispersion over the experimental spectral range. They analyzed the elastic scattering of a levitated particle at a scattering angle of $\pi / 2$ and used two detectors in the planes parallel and perpendicular to the electric field vector of the incident beam stemming from a scanning ring dye laser. Conceptually, our approach $(\mathrm{Te}, 2011)$ is similar, but we use a narrow bandwidth tunable diode laser (TDL, New Focus, model Velocity 6312 , tuning range $765-781 \mathrm{~nm}$, linewidth $(5 \mathrm{~s})<5 \mathrm{MHz})$ instead of a dye laser and only one detector (Si photo diode, New Focus femtowatt photo receiver, model 2151 ), also at a scattering angle of $\pi / 2$. At this angle, transverse electric (TE) mode resonances can be detected in the plane perpendicular to the plane of polarization and transverse magnetic (TM) mode resonances can be detected in the plane parallel to the plane of polarization (Huckaby et al., 1994). To observe both TE- and TM-mode resonances separately, we use a $\lambda / 2$-wave plate to rotate the polarization of the incoming beam and measure both polarizations sequentially. The detector samples the scattered light through the same optics that are used to collect the two-dimension angular scattering pattern. This has the advantage of measuring 


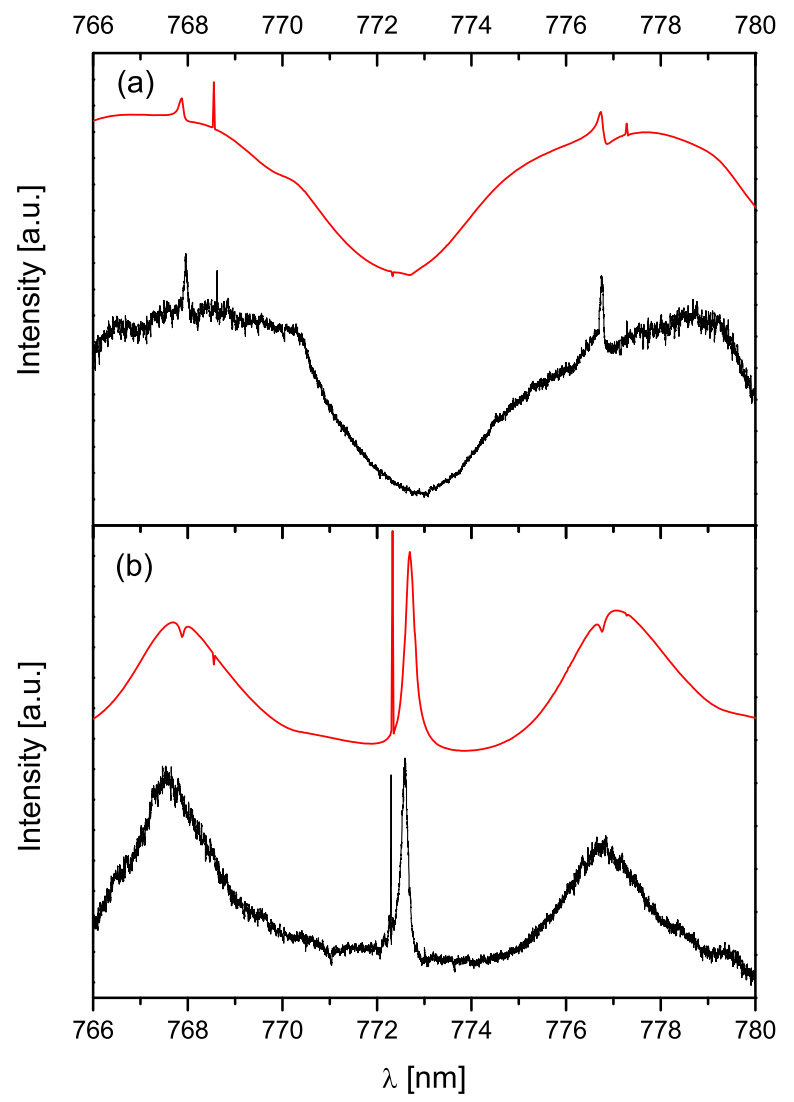

Figure 1. (a) TE and (b) TM spectra of shikimic acid particle under dry conditions at room temperature. Black line: experimental data. Red line: Mie calculation for a homogeneous particle with radius $7.756 \mu \mathrm{m}$ and refractive index of 1.540 .

both phase function and Mie resonance spectra over the same scattering angle range and the disadvantage of a rather large collection angle for the Mie resonance spectroscopy. To account for intensity modulation caused by weak etaloning of the laser during scan and by some residual interference at the windows of the chamber we use a second detector to monitor the intensity of the reflected incoming beam as reference.

To avoid being limited by the accuracy of the wavelength reported by the commercial laser (approximately $0.1 \mathrm{~nm}$ ) we use a custom-made wavemeter, following the design of Fox et al. (1999). As a reference laser we use a HeNe-laser (Stone et al., 2009), which is frequency stabilized using the design of Balhorn et al. (1972), yielding a relative uncertainty of the frequency stabilization of about 1 part in $10^{7}$. Overall, the precision of the wavemeter is of the order of a few parts in $10^{6}$, which was verified by measuring the hyperfine structure of the Rubidium $\mathrm{D}_{2}$ line (centered at $780.04 \mathrm{~nm}$ ), see Appendix A.

In the present application we need the high resolution spectroscopy only to measure the radius and the real part of the refractive index at a number of discrete relative humidities because we follow the radius change with the "white"- light resonance spectroscopy (see discussion in Sect. 3.2). Hence, we do analyze the spectra without any advanced fitting algorithm, but compare measured with calculated spectra manually, as shown in Fig. 1. For an initial guess of size we use the measured phase function and apply Chylek's approximation of the spacing between resonances (Chylek, 1976, 1990). We find the best fit in size at a fixed refractive index testing a reasonable range of refractive indices. In this way, we can easily determine the real part of the refractive index, $m_{\mathrm{TDL}}$, with an accuracy of better than 0.005 and a corresponding accuracy in size of about $2 \times 10^{-3} \mu \mathrm{m}$. This is sufficient for the type of experiments we are discussing here, since the limiting factor in the analysis of the data is the uncertainty in measured relative humidity. Since at least two resonances should be fitted, there is a lower limit in particle size of about $4-5 \mu \mathrm{m}$ for the tuning range of our laser. The spectra shown in Fig. 1 were recorded with a scanning speed of $0.04 \mathrm{~nm} \mathrm{~s}^{-1}$; i.e., a spectral scan took a little less than $6 \mathrm{~min}$. For a detailed discussion on the accuracy of aerosol refractive index retrieval from single particle techniques see Mason et al. (2012). As mentioned above, we continuously measure low resolution Mie spectra using the LED centered at the wavelength of the sodium D-Line $(589 \mathrm{~nm})$. These spectra are then used to track changes in the radius. Since the radius at specific relative humidities is now known from the concurrent high resolution measurements, the refractive index at the sodium D-Line needed for retrieval of these data, $m_{\mathrm{D}}$, can be determined by a Mie fit to the low resolution spectrum using dispersion, $m_{\mathrm{D}}>m_{\mathrm{TDL}}$, and the radius as a constraint.

\section{Measurement strategy and data analysis}

It has been shown previously that water diffusion constants can be deduced by combining stepwise changes in relative humidity with an accurate measurement of the response in size of a levitated particle (Zobrist et al., 2011; Bones et al., 2012; Lienhard et al., 2014). In these previous investigations, additional data were available (refractive indices, densities) helping to constrain the size retrieval. Here, we want to illustrate how we deduce the refractive index, density and water activity of an aerosol particle if such data are either completely lacking, or only available for a very limited concentration range. So far, we have restricted our measurements to low-volatility compounds to simplify the retrieval of composition from size and mass change.

\subsection{Retrieval of mass growth factor}

Traditionally, water activity data have been obtained utilizing the DC voltage compensating the gravitation force in the EDB (Tang and Munkelwitz, 1994; Zardini et al., 2008). As the exact charge of the particle is not known, only relative changes in mass can being inferred; a reference state 


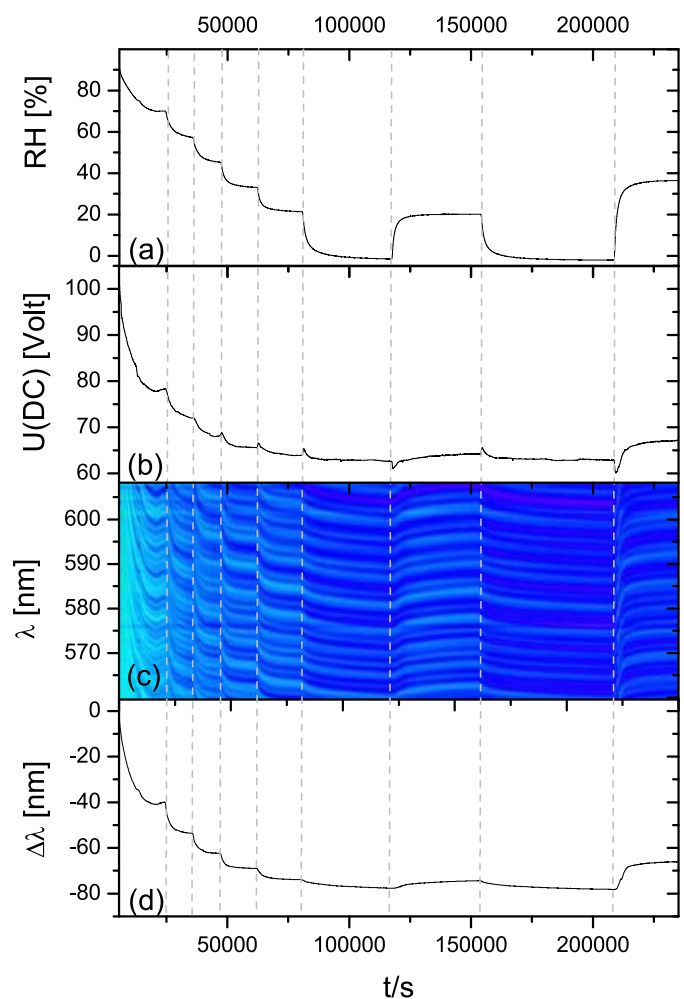

Figure 2. Typical experimental run on a shikimic acid particle with dry radius of $8.385 \mu \mathrm{m}$ at $T=293.5 \mathrm{~K}$. (a) Relative humidity measured close to the droplet. (b) DC voltage compensating gravitational force. (c) False color map of intensity of Mie resonance spectra measured with the LED (dark color low intensity, bright color high intensity). (d) Shift in wavelength $\Delta \lambda=\lambda(t)-\lambda_{0}$ of a resonance initially $(t=0)$ being observed at $\lambda_{0}=578.99 \mathrm{~nm}$. When this resonance leaves the spectral window, we continue by following another resonance as described by Zardini et al. (2006). Vertical dashed lines indicate humidity change.

is needed to calculate a mass growth factor. If bulk data cannot be obtained either because the amount of sample material is limited, or because solubility is very low, the voltage measured under dry conditions may serve as reference point. Figure 2 shows a typical experimental run performed at a constant temperature of $293.5 \mathrm{~K}$.

A closer look at the data of Fig. 2 shows that the accuracy of the voltage data is limited by spurious signals when a rapid change in relative humidity is applied. The DC voltage in our apparatus does not only compensate the gravitational force but also counteracts the Stokes force of the gas flow. Since the relative humidity is adjusted by changing the gas flow ratio of dry and humidified flow, very rapid changes lead to a response in the feedback loop adjusting the DC voltage, due to finite response of the mass flow controllers. This limits the accuracy of the voltage data to about $\pm 2 \mathrm{~V}$. The data in panel $d$ showing the shift in resonance wavelength are free from this artifact.

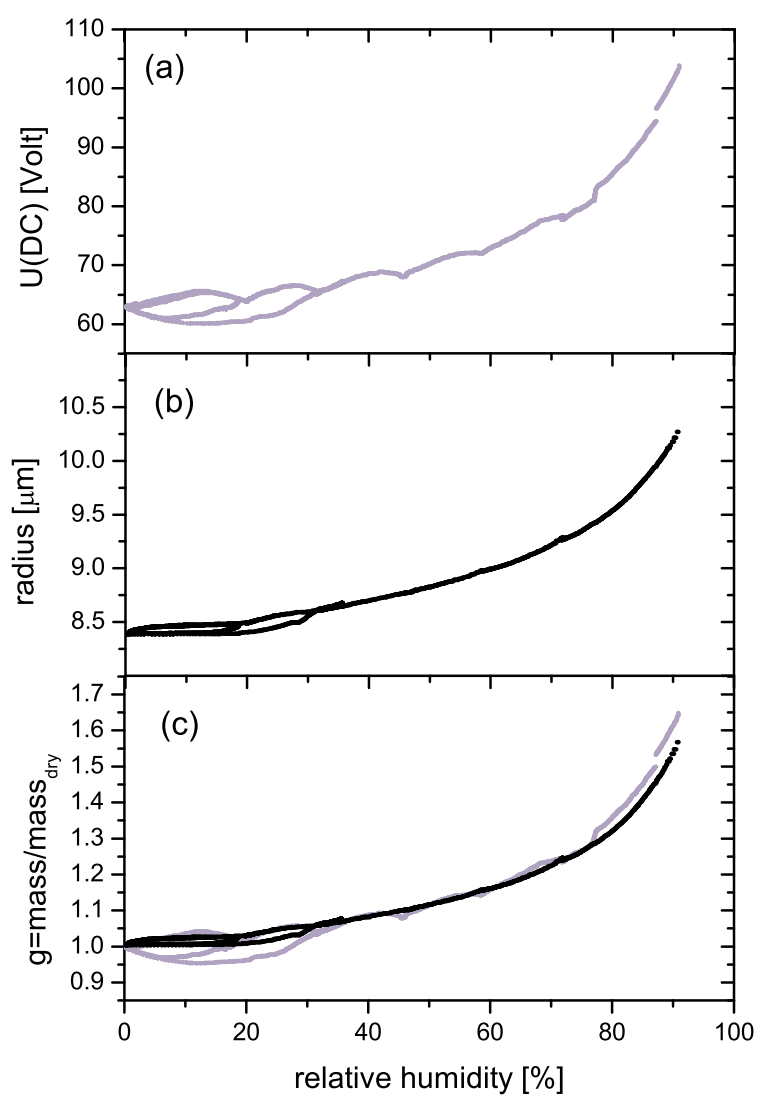

Figure 3. Data of Fig. 2 plotted vs. relative humidity. (a) DC voltage compensating gravitational force. (b) Radius data, see text on how to obtain those from the data of Fig. 2d. (c) Mass to dry mass ratio (growth factor $g$ ) calculated from both voltage (gray) and radius data (black).

For an aqueous aerosol particle with negligible vapor pressure of the solute(s) $\left(p_{\text {solute }} \leq 10^{-7} \mathrm{~Pa}\right)$ only water is exchanged between gas and particle phase. Under these conditions, the particle's mass growth factor can be directly inferred from the DC voltage compensating the gravitational force once the voltage under dry conditions has been measured. For aqueous organic systems, the kinetic uptake impedance due to slow water diffusion in semi-solid or glassy aerosol may substantially lengthen the time needed to reach thermodynamic equilibrium (whereas the transport of $\mathrm{H}_{2} \mathrm{O}$ molecules through gas phase by ventilation and molecular diffusion is very fast). This becomes evident when plotting the DC-voltage data of Fig. 2 vs. relative humidity as done in Fig. 3a and the inferred mass growth as in panel c. Below a relative humidity of about 0.3 the data show hysteresis loops, indicating delayed water uptake upon humidification and delayed water release upon drying (Zobrist et al., 2011). (Note that growth factors smaller than 1 derived from DC voltage data originate from the uncertainty of the measurement.) However, these are not caused by deliquescence and efflorescence (i.e., the crystallization of shikimic 
acid) but by kinetic limitations. Crystalline particles in the size range of our particles show deviations from spherical shape, which is easily detected in our setup when measuring the 2-dimensional angular optical scattering pattern (Krieger and Meier, 2011) or in fluctuations in the resonance spectra shown in Fig. 2c (Zardini and Krieger, 2009). We detected no sign of crystallization in our experiments even under the driest conditions.

In this study, we determine the equilibrium data (e.g., of growth factor) by considering the points where both branches of the loop overlap. Such overlap occurs either at higher RH, where equilibration within the particle phase is fast, or after prolonged waiting time at low RH. An important measure of the properties of the solution, which we measure here, is its water activity $a_{\mathrm{w}}=p_{\mathrm{H}_{2} \mathrm{O}}^{\mathrm{sol}}(T) / p_{\mathrm{H}_{2} \mathrm{O}}^{\mathrm{w}}(T)$, where $p_{\mathrm{H}_{2} \mathrm{O}}^{\text {sol }}$ is the $\mathrm{H}_{2} \mathrm{O}$ vapor pressure of the solution, and $p_{\mathrm{H}_{2} \mathrm{O}}^{\mathrm{W}}$ is the $\mathrm{H}_{2} \mathrm{O}$ vapor pressure of pure water, both at the same temperature. Under equilibrium conditions, the water activity $\left(a_{\mathrm{w}}\right)$ of the aqueous solution is equal to the measured relative humidity.

Note that the inverse of the mass growth factor $(g=$ $m(\mathrm{RH}) / m(\mathrm{RH}=0)=\left(m_{\mathrm{s}}+m_{\mathrm{H}_{2} \mathrm{O}}\right) / m_{\mathrm{s}}$, with $m_{\mathrm{s}}$ being the mass of the solute and $m_{\mathrm{H}_{2} \mathrm{O}}$ being the mass of water) is equal to the mass fraction of solute, $w_{\mathrm{s}}$, since the change in mass is only caused by water uptake. We will use mass fraction data to constrain the density as explained in the following section.

\subsection{Conversion of Mie resonance data to size and concentration}

In Sect. 2 we explained how we obtain size and refractive index at a fixed RH using the high resolution Mie resonance spectra. When the particle radius is $r_{0}$ at time $t_{0}$, the LED based resonance shift, $\Delta \lambda$, shown in Fig. $2 \mathrm{~d}$ can be used to calculate the radius at other times, $r(t)$. If the refractive index did not change with size, the radius were easily obtained by noting that the Mie parameter, $X$, of a specific resonance, $X_{0}=2 \pi r_{0} / \lambda_{0}$, stays constant, i.e., $X(t)=X_{0}$ (Zardini et al., 2006):

$r(t)=\frac{\left(\lambda_{0}+\Delta \lambda(t)\right) r_{0}}{\lambda_{0}}$.

However, when the radius change is accompanied by a change in composition, e.g., uptake of water by an aqueous particle, the refractive index of the particle will change as well. A change in the real part of the refractive index from $m_{\mathrm{D}, 0}$ to $m_{\mathrm{D}}(t)$ leads to an additional shift in the wavelength of a mode in the Mie resonance spectrum (Ray et al., 1991). The combined effect can be accounted for by solving iteratively Eq. (2) for the radius as a function of time:

$$
\begin{aligned}
r(t)= & \left\{1-K\left(m_{\mathrm{D}}(t), X\right) \frac{m_{\mathrm{D}}(t)-m_{\mathrm{D}, 0}}{m_{\mathrm{D}, 0}}\right\} \\
& \frac{\left(\lambda_{0}+\Delta \lambda(t)\right) r_{0}}{\lambda_{0}} .
\end{aligned}
$$

An iterative calculation is needed because of the implicit radius dependence of $m_{\mathrm{D}}(t)$. In general, the proportionality factor, $K$, depends on refractive index and Mie parameter, but Ray et al. (1991) have shown that $K\left(m_{\mathrm{D}}, X\right)$ varies between 0.94 and 0.96 for $1.30 \leq m_{\mathrm{D}} \leq 2.00$ when the Mie parameter, $X$, is of the order of 100 as is the case in our experiments. In this study, we take $K$ as a constant factor of 0.95 .

Neither the refractive index of the solute nor its concentration dependence in aqueous solution are known a priori. Our approach for retrieving the concentration dependence of refractive index is therefore as follows. The dependence of the refractive index on concentration in aqueous solution is well described using the molar refractivity of the solute (Born and Wolf, 1980); i.e., the refractive index is linear with solute molarity. Hence the refractive index, $m_{\mathrm{D}}\left(w_{\mathrm{s}}\right)$, as a function of mass fraction of solute, $w_{\mathrm{s}}$, is

$m_{\mathrm{D}}\left(w_{\mathrm{s}}\right)=m_{\mathrm{D}, \mathrm{H}_{2} \mathrm{O}}+\frac{\rho\left(w_{\mathrm{s}}\right)}{\rho_{\mathrm{s}}}\left(m_{\mathrm{D}, \mathrm{s}}-m_{\mathrm{D}, \mathrm{H}_{2} \mathrm{O}}\right) w_{\mathrm{s}}$,

with $m_{\mathrm{D}, \mathrm{s}}$ being the refractive index of the pure solute, $\rho_{\mathrm{s}}$ being the density of the pure solute and $\rho\left(w_{\mathrm{s}}\right)$ being the density of the aqueous solution at the concentration $w_{\mathrm{s}}$. To determine $m_{\mathrm{D}, \mathrm{s}}$, first the refractive index of an aqueous solution close to saturation concentration was measured using an Abbe type of refractometer at the sodium D-Line wavelength and the density at this concentration was measured using a pycnometer, see Fig. 4. Since shikimic acid is not very soluble in water (mole fraction at saturation 0.025), these data alone lead neither to accurate estimates of the density of the solute $\rho_{\mathrm{S}}$ (or the equivalent molar volume) nor to those of $m_{\mathrm{D}, \mathrm{s}}$ (or the molar refractivity). For other samples of interest the amount of material available may not be sufficient to perform these type of bulk experiments at all. Therefore, we measure a high resolution Mie resonance spectrum and use the concurrent LED spectrum as described in Sect. 2 at very dry conditions to determine the refractive indices, $m_{\mathrm{TDL}}$ and $m_{\mathrm{D}}$. This yields the refractive index, $m_{\mathrm{D}, \mathrm{s}}$, of the solute as we assume $w_{\mathrm{s}}=1$.

What remains to be determined is the density of the solution, $\rho\left(w_{\mathrm{s}}\right)$. We assume that the density of binary aqueous solutions can be adequately approximated using the density of each the pure solute and water and the conventional volume additivity rule:

$\rho\left(w_{\mathrm{s}}\right)=\left(\frac{1-w_{\mathrm{s}}}{\rho_{\mathrm{H}_{2} \mathrm{O}}}+\frac{w_{\mathrm{s}}}{\rho_{\mathrm{s}}}\right)^{-1}$,

with $\rho_{\mathrm{H}_{2} \mathrm{O}}$ the density of water, and $\rho_{\mathrm{s}}$ the density of the dry, amorphous solute. For a number of selected aqueous organic 

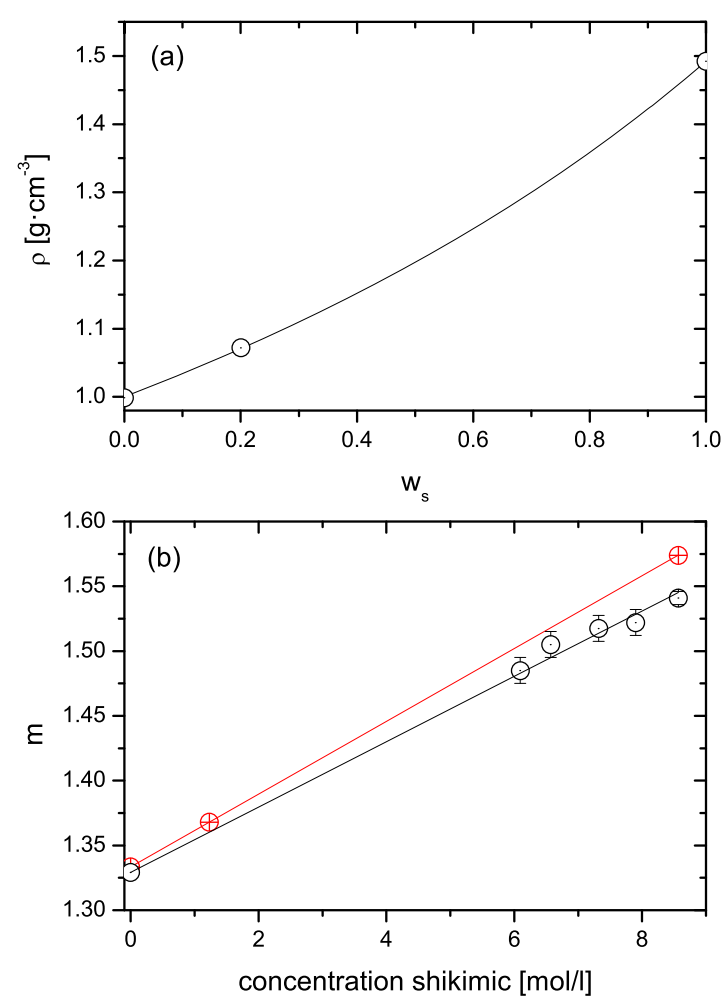

Figure 4. (a) Density vs. mass fraction parametrization of shikimic acid, the data point at $w=0.2001, \rho=1.072 \mathrm{~g} \mathrm{~cm}^{-3}$ was measured with a pycnometer, the density of the solute $\rho_{\mathrm{s}}=1.492 \mathrm{~g} \mathrm{~cm}^{-3}$ was determined as described in the text, the line is a plot of Eq. (4). (b) Refractive indices vs. molarity. $m_{\mathrm{TDL}}$ from high resolution Mie resonance spectra (black symbols), $m_{\mathrm{D}}$ (red symbols), lines are linear fits for the two different wavelengths to Eq. (3): $m_{\mathrm{TDL}, \mathrm{H}_{2} \mathrm{O}}=$ $1.3292, m_{\mathrm{TDL}, \mathrm{s}}=1.541, m_{\mathrm{D}, \mathrm{H}_{2} \mathrm{O}}=1.3334, m_{\mathrm{D}, \mathrm{s}}=1.574$.

mixtures (e.g., citric acid) this approximation proved to be accurate within $1 \%$ of the data (Lienhard et al., 2012).

The density of the pure solute, $\rho_{\mathrm{s}}$, is the only remaining unknown. To determine it we start using an initial guess, which allows us to calculate the refractive index $m_{\mathrm{D}}$ for all concentrations using Eqs. (4) and (3). Then $r(t)$ is computed solving Eq. (2). The mass growth factor can be calculated using the corresponding density and compared to the measured data shown in Fig. 3c. If mass growth from the DCvoltage data do not agree with the mass growth calculated from the spectra, we update the initial guess of $\rho_{\mathrm{S}}$ and calculate again until the agreement is satisfactory. For aqueous shikimic acid solutions this leads to densities and refractive indices at a temperature of $293.5 \mathrm{~K}$ as shown in Fig. 4. In addition to measuring the refractive index at very dry conditions, we measured the refractive index at several humidities as shown in panel $b$. These measurements support the value of the refractive index of the pure solute, $m_{\mathrm{TDL}, \mathrm{s}}$, and through Eq. (3), also the density of the pure solute, $\rho_{\mathrm{s}}$.

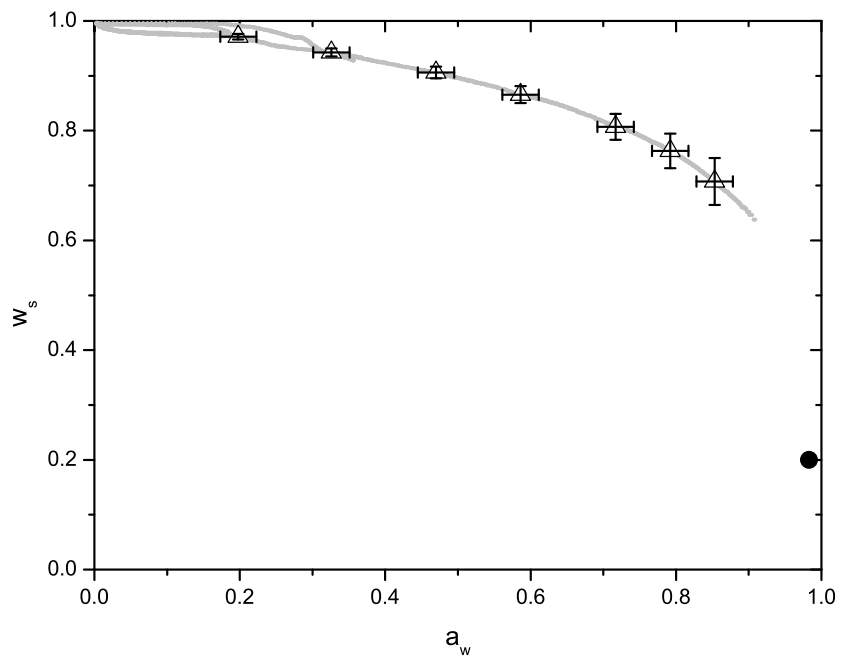

Figure 5. Mass growth data retrieved from radius measurements of Fig. $3 \mathrm{c}$ plotted as mass fraction of solute vs. $a_{\mathrm{w}}$ (gray line). The black circle marks a bulk data point of the saturated solution. We pick some discrete data points at humidities at which the particle is in equilibrium with the gas phase (open triangles) for fitting.

Note that we implicitly assume the particle to be homogeneous in the analysis outlined above. If there is a gradient in concentration within a particle due to kinetic uptake limitations, its refractive index will show a corresponding gradient. Strictly, Eq. (2) is no longer valid and a numerical modeling of the Mie resonance spectra of the inhomogeneous particle is needed to calculate the radius. However, the difference between the exact modeled results and those generated by applying Eq. (2) to an inhomogeneous particle is small compared to the uncertainty in our experiments (mainly related to the accuracy of relative humidity probe) (Lienhard et al., 2014).

\subsection{Parametrization of water activity}

From the type of data shown in Fig. 3c we derive a parametrization of water activity vs. concentration by noting that the inverse of the mass growth factor is equal to the mass fraction of solute $\left(w_{\mathrm{s}}\right)$ provided that only water is partitioning between gas and particle phase. The data of Fig. 3c are replotted as $w_{\mathrm{s}}$ vs. $a_{\mathrm{w}}$ in Fig. 5. From these data we select discrete data points at humidities at which the particle is in equilibrium with the gas phase by assuring that the same $w_{\mathrm{s}}$ was measured upon humidifying and drying. Such data were measured at $293.5 \mathrm{~K}$ for three different particles injected from fresh solutions. In addition, we used a commercial water activity meter (AquaLab, Model 3TE, Decagon Devices, USA) to measure the $a_{\mathrm{w}}$ close to a saturated solution. As can be seen in Figs. 5 and 6, this data point does not significantly constrain the parametrization because the water activity of the saturated solution is very close to that of pure water. 

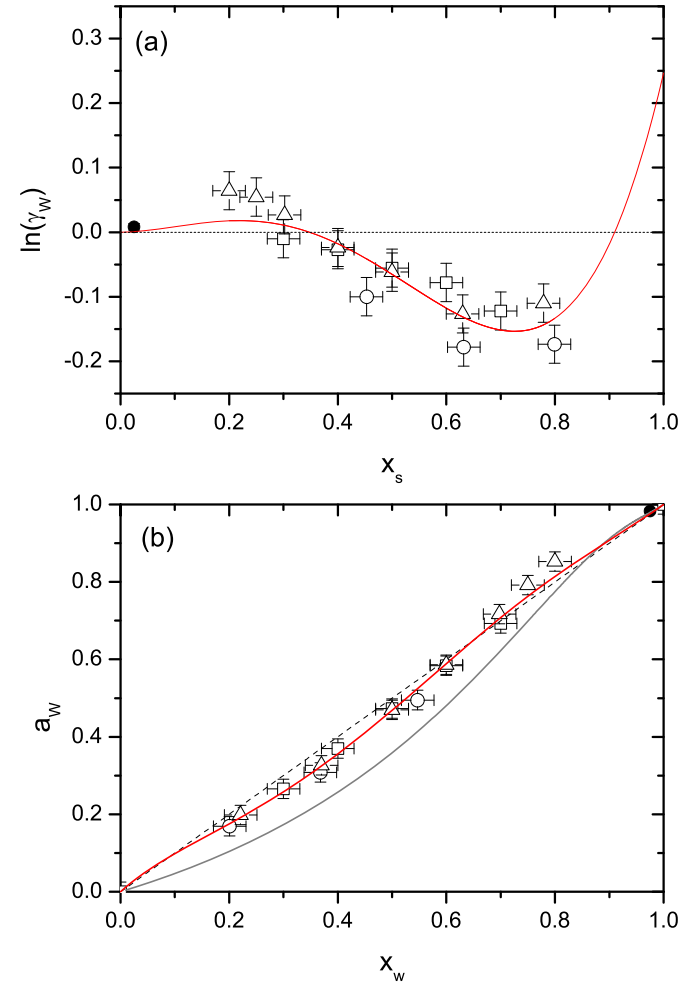

Figure 6. (a) Natural logarithm of water activity coefficient $\gamma_{\mathrm{w}}$ vs. mole fraction of shikimic acid, symbols are data for three independent particles, solid circle is the bulk data point, dashed line represents ideal behavior (Raoult's law), red line is the fit of Eq. (5) to the data. (b) The same data plotted as water activity vs. mole fraction of water, the gray line is the prediction of the thermodynamic model AIOMFAC (Zuend et al., 2011).

We convert $w_{\mathrm{s}}$ to mole fraction, $x_{\mathrm{s}}$, and obtain the water activity coefficients, $\gamma_{\mathrm{w}}$, according to

$\ln \gamma_{\mathrm{w}}=x_{\mathrm{s}}^{2}\left\{(E+3 F+5 G)-(4 F+16 G) x_{\mathrm{s}}+12 G x_{\mathrm{s}}^{2}\right\}$

(McGlashan, 1963), where $E, F, G$ are fitting parameters (see Fig. 6).

The advantage of this approach is that once the fitting parameters are determined, it allows the direct calculation of the activity coefficient of the solute, $\gamma_{\mathrm{s}}$, via the DuhemMargules relations as (McGlashan, 1963):

$\ln \gamma_{\mathrm{s}}=x_{\mathrm{w}}^{2}\left\{(E+F+G)-(4 F+8 G) x_{\mathrm{w}}+12 G x_{\mathrm{w}}{ }^{2}\right\}$.

For shikimic acid, we found $E=-0.182, F=-0.079$ and $G=0.349$. It is evident from Fig. 6 that the water activity of aqueous shikimic acid solutions deviate only slightly from ideal behavior (Raoult's law). The prediction by the thermodynamic model AIOMFAC (Zuend et al., 2011), namely lower $a_{\mathrm{w}}$ than ideal for high concentrations of shikimic acid and crossing to higher $a_{\mathrm{w}}$ than ideal at low shikimic acid concentration, is in agreement with the data.

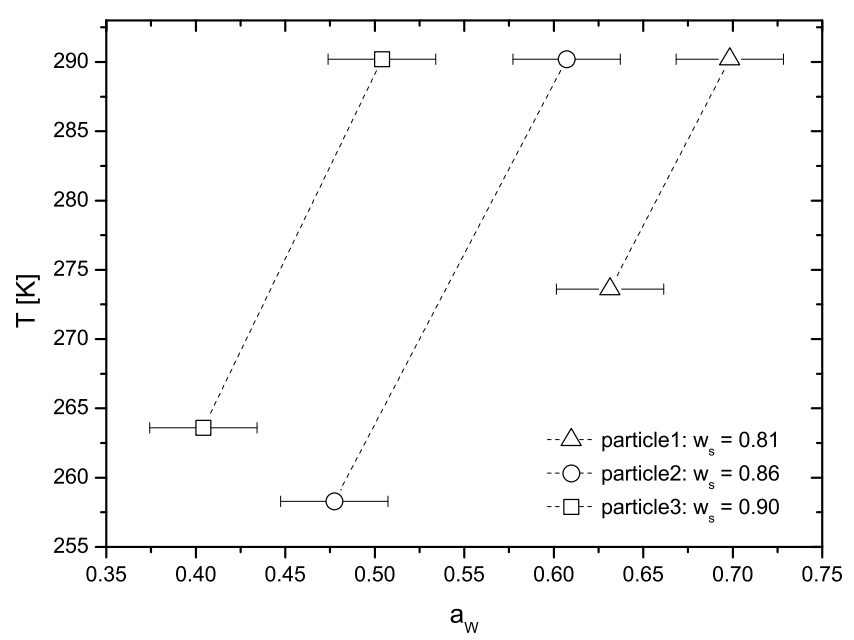

Figure 7. Temperature vs. $a_{\mathrm{w}}$ for three different shikimic acid particles at fixed concentrations (as indicated in the legend). The dashed lines serve only as a guide to the eye.

However, the magnitude of the deviation is smaller than predicted and the crossing is at higher concentration of shikimic acid than predicted.

We did not study the temperature dependence of $a_{\mathrm{w}}$ systematically, but obtained some data with known concentration at low temperatures at which the particles where equilibrated for a long time. We take the particle as equilibrated if the growth factor at a particular relative humidity is the same upon humidification and drying. The time required depends on both, RH and temperature. Our data suggest a positive slope of $\mathrm{d} a_{\mathrm{w}} / \mathrm{d} T$ of about $4 \times 10^{-3} \mathrm{~K}^{-1}$, see Fig. 7. Since our uncertainty in RH is almost as large as the shift in $a_{\mathrm{w}}$ over the temperature range considered in this study, we do not correct for the temperature dependence of $a_{\mathrm{w}}$ when analyzing the kinetic data at low temperatures.

\subsection{Analysis of kinetic data}

Having characterized the thermodynamics of aqueous shikimic acid, we will proceed with analyzing the kinetic behavior, which is apparent in the raw data of Fig. 3 as hysteresis loops (cf. discussion in Zobrist et al., 2011). In Sect. 3.4.1 we briefly summarize the numerical diffusion model used for analysis and report water diffusivity as a function of temperature and concentration in Sect. 3.4.2.

\subsubsection{Diffusion model}

In aqueous organic solutions, water acts as a plasticizer (Koop et al., 2011), and hence the water diffusion coefficient becomes concentration dependent. We solve the diffusion equation in spherical coordinates to retrieve concentration dependent water diffusion coefficients. Since our approach has been already described in depth by Zobrist et al. (2011), we will give only a brief summary of the underlying 

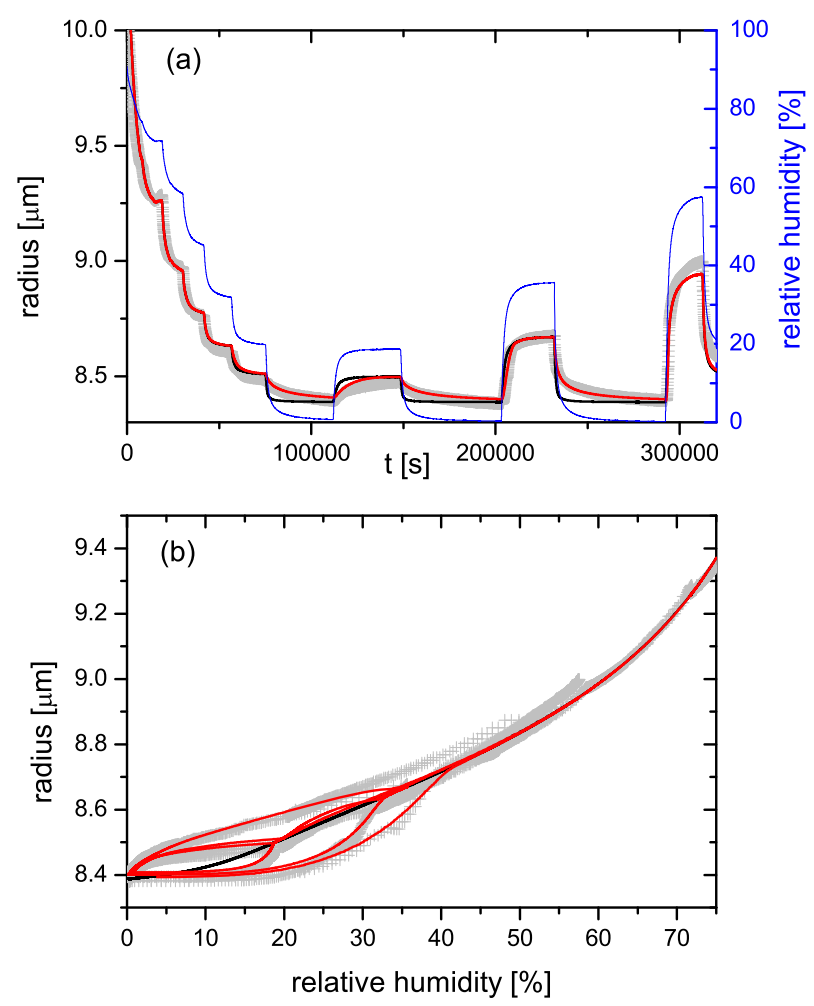

Figure 8. (a) Data of Fig. 2 converted to radius vs. time (gray crosses), equilibrium prediction of radius using the activity parametrization of Sect. 3.3 (black line), diffusion model with a diffusivity of $\log \left(D_{\mathrm{H}_{2} \mathrm{O}}\right)=-8.7-7.5\left(1-a_{\mathrm{w}}\right)$ using the same activity parametrization (red line). Relative humidity (blue line) right axis. (b) The same data plotted vs. relative humidity. At $\mathrm{RH}>40 \%$ at 293.5 K we observe no kinetic limitations to water uptake or release, while below that humidity kinetic limitations become clearly visible by the hysteresis loops in water uptake and release.

principles. The diffusion equation is

$$
\begin{aligned}
\frac{\partial n}{\partial t} & =\nabla\left(D_{\mathrm{H}_{2} \mathrm{O}}(n, T) \nabla n\right) \\
& =\frac{1}{r^{2}} \frac{\partial}{\partial r}\left(r^{2} D_{\mathrm{H}_{2} \mathrm{O}}(n, T) \frac{\partial n}{\partial r}\right),
\end{aligned}
$$

where $n$ is the number density of water molecules in the particle, $t$ is the time, and $r$ is the distance from the particle center. Due to the concentration dependence of $D_{\mathrm{H}_{2} \mathrm{O}}$, the diffusion equation becomes non-linear. This leads to steep diffusion fronts instead of the more commonly known creeping diffusion tails (Crank, 1975). It also means that a general analytical solution for Eq. (7) cannot be found, and the problem instead needs to be solved numerically. Our numerical model separates the particle into up to several thousands of individual shells. Growth and shrinkage of the particle are then the result of water diffusion between those shells. The change of the number of water molecules, $\Delta N_{i}$, within the shell $i$ is described by the following:

$\Delta N_{i}=\left(f_{i-\frac{1}{2}}-f_{i+\frac{1}{2}}\right) \Delta t$,

where $f_{i-\frac{1}{2}}$ is the flux of water molecules from shell $i-1$ to shell $i$, whereas $f_{i+\frac{1}{2}}$ describes the flux of water molecules from shell $i$ to shell $i+1$. The time interval, $\Delta t$, is chosen so that $\Delta N_{i}$ does not vary by more than a specified amount (usually $\leq 2 \%$ ) within one time step. Shell thickness was adjusted dynamically to enable resolution of steep gradients when necessary. However, a minimum thickness of $0.3 \mathrm{~nm}$ was chosen to represent the size of a water molecule. Note that the surface layer of the particle (i.e., the outermost shell) is kept in equilibrium with the gas phase at all times so that mass transport is never limited by gas-phase diffusion. While gas phase diffusion under our experimental condition is indeed significantly faster than liquid phase diffusion (Zobrist et al., 2011), recent experiments have shown a difference between adsorption and desorption at low humidity (Lienhard et al., 2014). These findings question the assumption of the outermost shell always being in thermodynamic equilibrium, but this does not alter the derived diffusion coefficients significantly (Lienhard et al., 2014).

\subsubsection{Water diffusivity parametrization of aqueous shikimic acid}

To obtain water diffusivity from data as shown in Fig. 2, we first calculate radius vs. time as explained in Sect. 3.2 and then run the numerical model with different test dependencies of $\log D_{\mathrm{H}_{2} \mathrm{O}}$ vs. $a_{\mathrm{w}}$ until the fit appeared satisfactory upon manual inspection. An example of the fit to the data of Fig. 2 is shown in Fig. 8.

As in Fig. 8 a typical experiment at constant temperature covers a certain range of $a_{\mathrm{w}}$ where deviations from instantaneous equilibration are detectable. For a global fit of diffusivity we extract data points roughly spaced 0.15 in water activity within the humidity range covered by the specific experiment. The diffusivities at all investigated temperatures and concentrations are shown in Fig. 9. We performed measurements between 294 and $251 \mathrm{~K}$ and from dry conditions to about $80 \%$ RH. However, above about $50 \%$ RH it is not possible to extract diffusion coefficients with our setup because the rate of humidity change with time has an upper limit. This mainly due to limitations in gas flow because higher flow rates affect the stability of the particle in the EDB.

Analogous to Lienhard et al. (2014), we use a modified Vignes-type equation (Vignes, 1966) to empirically fit these data:

$D_{\mathrm{H}_{2} \mathrm{O}}=\left(D_{\mathrm{H}_{2} \mathrm{O}}^{0}\right)^{x_{\mathrm{W}} \alpha}\left(D_{\mathrm{s}}^{0}\right)^{1-x_{\mathrm{w}} \alpha}$,

where $D_{\mathrm{H}_{2} \mathrm{O}}^{0}$ and $D_{\mathrm{s}}^{0}$ are the diffusion coefficients for water in pure water and pure shikimic acid, respectively. Their 
temperature dependence can be fitted to a Vogel-FulcherTammann expression:

$\log _{10}\left(D_{\mathrm{H}_{2} \mathrm{O}}^{0}\right)=-6.514-\frac{387.4}{T-118}$,

and

$\log _{10}\left(D_{\mathrm{s}}^{0}\right)=-9.35-\frac{542.8}{T-211}$,

where the numbers for $D_{\mathrm{H}_{2} \mathrm{O}}^{0}$ are taken from Smith and Kay (1999) and the units for the temperature is $\mathrm{K}_{\text {and }} \mathrm{m}^{2} \mathrm{~s}^{-1}$ for the diffusion coefficient. To be consistent with our previous work (Lienhard et al., 2014), we introduce an empirical correction parameter $\alpha$, having the form of an activity coefficient:

$\ln (\alpha)=\left(1-x_{\mathrm{w}}\right)^{2}\left[C+3 D-4 D\left(1-x_{\mathrm{w}}\right)\right]$,

where $C$ and $D$ are temperature dependent:

$C=-6.55+0.025 T$,

$D=7.122-0.0261 T$,

with $C(T>258 \mathrm{~K})=C(T=258 \mathrm{~K})$ and $D(T>273 \mathrm{~K})=$ $D(T=273 \mathrm{~K})$.

The resulting fit to Eq. (9) with $\alpha$ calculated according to Eq. (12) is shown in Fig. 9. The correction parameter $\alpha$ stays close to 1 in this fit for the entire temperature and concentration range. Therefore, we plot in Fig. 9 also the contours for the different temperatures without correction (i.e., $\alpha=1$ ). This leads to significant differences only for temperatures below $258 \mathrm{~K}$. However, as only few data are available in this temperature range and the fits are just starting to diverge, measurements at even lower temperatures would be helpful for a more thorough comparison of the two fits. We conclude that the three parameter fit without correction term describes the water diffusivity satisfactorily.

Figure 9 also show water diffusivity in aqueous sucrose (Zobrist et al., 2011) for the highest and lowest temperature measured here. While the logarithm of diffusivity of water increases with water activity almost linearly in shikimic acid, sucrose shows less increase under dry conditions. In general the diffusivity is smaller in sucrose, but at all temperatures the diffusivity agrees within 1 order of magnitude for dry conditions (up to a water activity of about 0.25 ) and deviates at most by 2 orders (at water activity of about 0.5 ). Further investigations are needed to determine whether water diffusivity in aqueous organic compounds behaves similarly with temperature and water activity for different compounds and to which degree a model compounds like shikimic acid may serve as a proxy for secondary organic aerosol.

\section{Conclusions}

We have presented a measurement and data retrieval technique to extract water activity, density, refractive index and

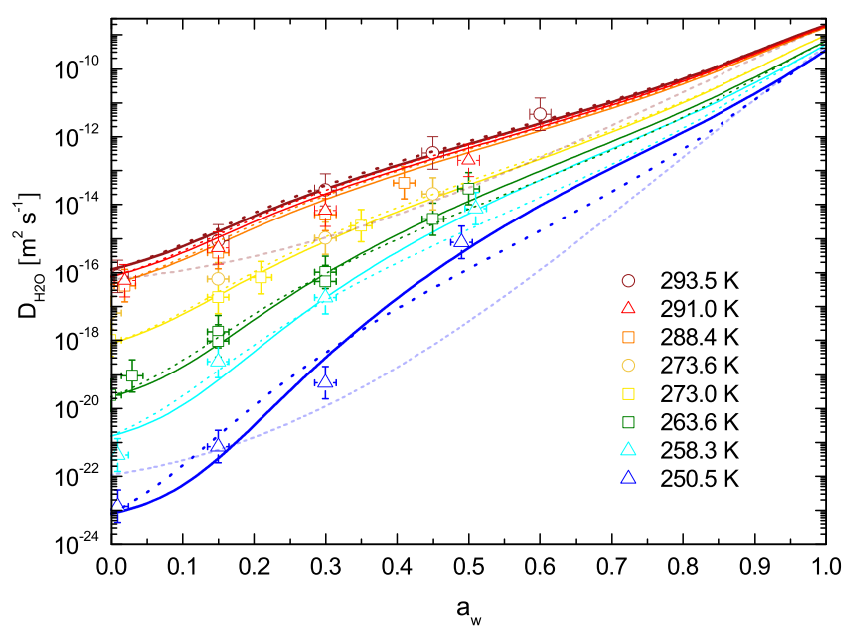

Figure 9. $D_{\mathrm{H}_{2} \mathrm{O}}$ as a function of $a_{\mathrm{W}}$ for the investigated temperatures indicated by different colors. Different symbols represent different particles. The lines represent fits to Eq. (9). Solid lines were fitted with an $\alpha$ calculated according to Eq. (12), dotted lines with $\alpha=1$. For comparison, the faint, short, dashed lines in corresponding colors show the parametrization of $D_{\mathrm{H}_{2} \mathrm{O}}$ for sucrose of Zobrist et al. (2011) for 250.5 and $293.5 \mathrm{~K}$.

water diffusion constants from mass-to-charge data and light scattering data of single levitated droplets in an electrodynamic balance. In particular, we have shown that an iterative procedure combining mass-to-charge data with Mie resonance spectroscopy yields robust data for parametrizing activity as well as water diffusivity. If solubility and amount of material available allow for bulk measurements of water activity, density and refractive index, these measurements provide constrains for simple mixing rules and hence further increase the accuracy of the parametrizations. However, even if measurements in the bulk are not available or possible, the technique presented here allows to constrain parametrizations well enough to be of use for atmospheric applications. We plan to use it to characterize secondary organic aerosol, which was collected on filters during oxidation experiments in which only about a milligram of material is available.

We studied aqueous shikimic acid aerosol in detail, a model system for aged, oxygenated organic aerosol. Due to its single carbon-carbon double bond it is especially well suited for heterogeneous chemistry studies because the consumption of this double bond can be easily monitored with different spectroscopic techniques. Our study shows that its activity in aqueous solution is close to ideal; i.e., it almost follows Raoult's law. In addition, the water diffusivity in aqueous shikimic acid turns out to closely follow the simple empirical Vignes equation in contrast to other binary systems, e.g., aqueous citric acid. Comparison with secondary organic aerosol samples in the future will show how well shikimic acid represents the physical and chemical properties of atmospheric organic aerosol. 


\section{Appendix A: Hyperfine spectrum of Rubidium}

To determine the accuracy and precision of the homemade wavemeter we measured the hyperfine structure of the $\mathrm{Ru}$ bidium $\mathrm{D}_{2}$ line. The laser emission of the TDL is split into two beams by a $50 / 50$ beam splitter. One beam is focused into a Rubidium glass cell, and the other beam is fed into the Michelson interferometer to perform the wavelength measurement. A photodetector (New Focus Visible Femtowatt Photoreceiver, model 2151) is used to detect the fluorescence of the excited Rubidium. The fluorescence signal and the wavelength measurements are simultaneously recorded; the results are shown in Fig. A1.

The measured wavelength deviate from literature data by no more than $0.001 \mathrm{~nm}$.

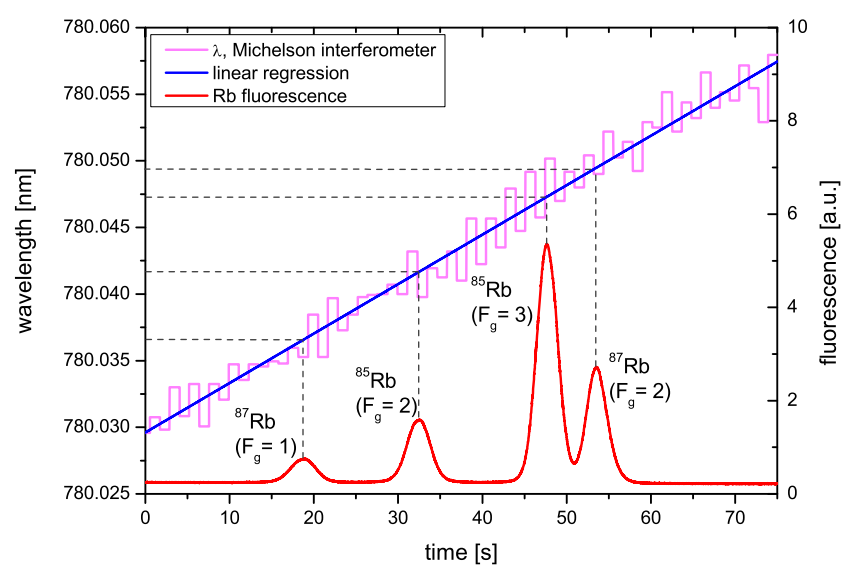

Figure A1. Rubidium spectroscopy using the piezoelectrically tuned TDL (780.030-780.060 nm with $0.0004 \mathrm{~nm} \mathrm{~s}^{-1}$ scan speed). The red line depicts the fluorescence light intensity and the violet line the Michelson interferometer wavelength measurement. The gray dashed lines denote the measured wavelengths needed to excite the $\mathrm{D}_{2}$ transitions. With the resolution of our experiment the hyperfine splitting of the level $5 \mathrm{P}_{3 / 2}$ is not resolved, while the numbers $F_{g}$ denote the hyperfine levels of $5 \mathrm{~S}_{1 / 2}$. 
Acknowledgements. We acknowledge Andreas Zuend for calculating the AIOMFAC prediction of Fig. 6 and Claudia Marcolli for helpful discussions. This work was supported by the ETH Research Grant ETH-0210-1 and by the Swiss National Science Foundation under project no. P2EZP2-152205. S. S. Steimer and M. Ammann acknowledge support by the EU FP7 project PEGASOS and by the University of Bern.

Edited by: F. Pope

\section{References}

Ashkin, A. and Dziedzic, J. M.: Observation of resonances in the radiation pressure on dielectric spheres, Phys. Rev. Lett., 38, 13511354, 1977.

Balhorn, R., Kunzmann, H., and Lebowsky, F.: Frequency stabilization of internal-mirror helium-neon lasers, Appl. Optics, 11, 742-744, 1972.

Bones, D. L., Reid, J. P., Lienhard, D. M., and Krieger, U. K.: Comparing the mechanism of water condensation and evaporation in glassy aerosol, P. Natl. Acad. Sci. USA, 109, 11613-11618, 2012

Born, M. and Wolf, E.: Principles of Optics, 6th edn., Pergamon Press, Oxford, UK, 1980.

Budavari, B. (Ed.): The Merck Index: an Encyclopedia of Chemicals, Drugs, and Biologicals, 12th Edn., Merck \& Co., Whitehouse Station, USA, 1458, 1996

Chylek, P.: Partial-wave resonances and the ripple structure in the Mie normalized extinction cross section, J. Opt. Soc. Am., 66, 285-287, 1976.

Chylek, P.: Resonance structure of Mie scattering: distance between resonances, J. Opt. Soc. Am. A, 7, 1609-1613, 1990.

Chylek, P., Ramaswamy, V., Ashkin, A., and Dziedzic, J. M.: Simultaneous determination of refractive index and size of spherical dielectric particles from light scattering data, Appl. Optics, 22, 2302-2307, 1983.

Colberg, C. A., Krieger, U. K., and Peter, Th.: Morphological investigations of single levitated $\mathrm{H}_{2} \mathrm{SO}_{4} / \mathrm{NH}_{3} / \mathrm{H}_{2} \mathrm{O}$ aerosol particles during deliquescence/efflorescence experiments, J. Phys. Chem. A, 108, 2700-2709, 2004

Conwell, P. R., Rushforth, C. K., Benner, R. E., and Hill, S. C.: Efficient automated algorithm for the sizing of dielectric microspheres using the resonance spectrum, J. Opt. Soc. Am. A, 1, 1181-1187, 1984.

Crank, J.: The Mathematics of Diffusion, Clarendon Press, Oxford, 1975.

Fox, P. J., Scholten, R. E., Walkiewicz, M. R., and Drullinger, R. E.: A reliable, compact, and low-cost Michelson wavemeter for laser wavelength measurement, Am. J. Phys., 67, 624-630, 1999.

Gucker, F. T. and Rowell, R. L.: The angular variation of light scattered by single dioctyl phthalate aerosol droplets, Discuss. Faraday Soc., 30, 185-191, 1960.

Huckaby, J. L., Ray, A. K., and Das, B.: Determination of size, refractive index, and dispersion of single droplets from wavelength-dependent scattering spectra, Appl. Optics, 33, 7112-7125, 1994.

Koop, T., Bookhold, J., Shiraiwa, M., and Pöschel, U.: Glass transition and phase state of organic compounds: dependency on molecular properties and implications for secondary organic aerosols in the atmosphere, Phys. Chem. Chem. Phys., 13, 19238-19255, 2011.

Krieger, U. K. and Meier, P.: Observations and calculations of twodimensional angular optical scattering (TAOS) patterns of a single levitated cluster of two and four microspheres, J. Quant. Spectrosc. Ra., 112, 1761-1765, 2011

Krieger, U. K., Colberg, C. A., Weers, U., Koop, T., and Peter, T. Supercooling of single $\mathrm{H}_{2} \mathrm{~S}_{4} / \mathrm{H}_{2} \mathrm{O}$ aerosols to $158 \mathrm{~K}$ : no evidence for the occurrence of the octahydrate, Geophys. Res. Lett., 27, 2097-2100, 2000.

Kuwata, M. and Martin, S. T.: Phase of atmospheric secondary organic material affects its reactivity, P. Natl. Acad. Sci. USA, 109, 17354-17359, 2012.

Lienhard, D. M., Bones, D. L., Zuend, A., Krieger, U. K., Reid, J. P., and Peter, T.: Measurements of thermodynamic and optical properties of selected aqueous organic and organic-inorganic mixtures of atmospheric relevance, J. Phys. Chem. A, 116, 9954 9968, 2012.

Lienhard, D. M., Huisman, A. J., Bones, D. L., Te, Y.-F., Luo, B. P., Krieger, U. K., and Reid, J. P.: Retrieving the translational diffusion coefficient of water from experiments on single levitated aerosol droplets, Phys. Chem. Chem. Phys., 16, 16677-16683, 2014.

Mason, B. J., King, S.-J., Miles, R. E. H., Manfred, K. M., Rickards, A. M. J., Kim, J., Reid, J. P., and Orr-Ewing, A. J.: Comparison of the accuracy of aerosol refractive index measurements from single particle and ensemble techniques, J. Phys. Chem. A, 116, 8547-8556, 2012.

McGlashan, M. L.: Deviations from Raoult's law, J. Chem. Educ., 40, 516-518, 1963.

Medeiros, P. M. and Simoneit, B. R. T.: Source profiles of organic compounds emitted upon combustion of green vegetation from temperate climate forests, Environ. Sci. Technol., 42, 8310 8316, 2008.

Millikan, R. A.: Electrons ( + and - ), Protons, Photons, Neutrons and Cosmic Rays, Cambridge University Press, Cambridge, UK, 1935.

Moise, T. and Rudich, Y.: Reactive uptake of ozone by aerosolassociated unsaturated fatty acids: kinetics, mechanism, and products, J. Phys. Chem. A, 106, 6469-6476, 2002.

Preston, T. C. and Reid, J. P.: Accurate and efficient determination of the radius, refractive index, and dispersion of weakly absorbing spherical particle using whispering gallery modes, J. Opt Soc. Am. B, 30, 2113-2122, 2013.

Ray, A. K., Soury, A., Davis, E. J., and Allen, T. M.: Precision of light scattering techniques for measuring optical parameters of microspheres, Appl. Optics, 30, 3974-3983, 1991.

Shiraiwa, M., Ammann, M., Koop, T., and Pöschl U.: Gas uptake and chemical aging of semisolid organic aerosol particles, P. Natl. Acad. Sci. USA, 108, 11003-11008, 2011.

Smith, R. S. and Kay, B. D.: The existence of supercooled liquid water at 150 K, Nature, 398, 788-791, 1999.

Steimer, S. S., Lampimäki, M., Coz, E., Grzinic, G., and Ammann, M.: The influence of physical state on shikimic acid ozonolysis: a case for in situ microspectroscopy, Atmos. Chem. Phys., 14, 10761-10772, doi:10.5194/acp-14-10761-2014, 2014

Stone, J. A., Decker, J. E., Gill, P., Juncar, P., Lewis, A., Rovera, G. D., and Viliesid, M.: Advice from the CCL on the use 
of unstabilized lasers as standards of wavelength: the heliumneon laser at $633 \mathrm{~nm}$, Metrologia, 46, 11-18, 2009.

Tang, I. N. and Munkelwitz, H. R.: Simultaneous determination of refractive index and density of an evaporating aqueous solution droplet, Aerosol Sci. Tech., 15, 201-207, 1991.

Tang, I. N. and Munkelwitz, H. R.: Water activities, densities, and refractive indices of aqueous sulfates and sodium nitrate droplets of atmospheric importance, J. Geophys. Res., 99, 18801-18808, 1994.

Te, Y.-F.: Mie resonance spectroscopy of single levitated particle, M.S. Thesis, University of Zurich, available at: http: //www.physik.uzh.ch/groups/schilling/bama/MATe.pdf (last access: 19 January 2015), 2011.

Vignes, A.: Diffusion in binary solutions. Variation of diffusion coefficient with composition, Ind. Eng. Chem. Fund., 5, 189-199, 1966.

Virtanen, A., Joutsensaari, J., Koop, T., Kannosto, J., Yli-Pirilä, P., Leskinen, J., Mäkelä, J. M., Holopainen, J. K., Pöschl, U., Kulmala, M., Worsnop, D. R., and Laaksonen, A.: An amorphous solid state of biogenic secondary organic aerosol particles, Nature, 467, 824-827, 2010.

Zardini, A. A. and Krieger, U. K.: Evaporation kinetics of a nonspherical, levitated aerosol particle using optical resonance spectroscopy for precision sizing, Opt. Express, 17, 4659-4669, 2009.

Zardini, A. A., Krieger, U. K., and Marcolli, C.: White light Mie resonance spectroscopy used to measure very low vapor pressures of substances in aqueous solution aerosol particles, Opt. Express, 14, 6951-6962, 2006.
Zardini, A. A., Sjogren, S., Marcolli, C., Krieger, U. K., Gysel, M., Weingartner, E., Baltensperger, U., and Peter, T.: A combined particle trap/HTDMA hygroscopicity study of mixed inorganic/organic aerosol particles, Atmos. Chem. Phys., 8, 55895601, doi:10.5194/acp-8-5589-2008, 2008.

Zhou, S., Shiraiwa, M., McWhinney, R. D., Pöschl, U., and Abbatt, J. P. D.: Kinetic limitations in gas-particle reactions arising from slow diffusion in secondary organic aerosol, Faraday Discuss., 165, 391-406, 2013.

Zobrist, B., Marcolli, C., Pedernera, D. A., and Koop, T.: Do atmospheric aerosols form glasses?, Atmos. Chem. Phys., 8, 52215244, doi:10.5194/acp-8-5221-2008, 2008.

Zobrist, B., Soonsin, V., Luo, B. P., Krieger, U. K., Marcolli, C., Peter, T., and Koop, T.: Ultra-slow water diffusion in aqueous sucrose glasses, Phys. Chem. Chem. Phys., 13, 3514-3526, 2011.

Zuend, A., Marcolli, C., Booth, A. M., Lienhard, D. M., Soonsin, V., Krieger, U. K., Topping, D. O., McFiggans, G., Peter, T., and Seinfeld, J. H.: New and extended parameterization of the thermodynamic model AIOMFAC: calculation of activity coefficients for organic-inorganic mixtures containing carboxyl, hydroxyl, carbonyl, ether, ester, alkenyl, alkyl, and aromatic functional groups, Atmos. Chem. Phys., 11, 9155-9206, doi:10.5194/acp11-9155-2011, 2011. 